\title{
Channel Parameter Estimation for Scatter Cluster Model Using Modified MUSIC Algorithm
}

\author{
Jinsheng Yang, Xuzhao Wu, and Qing Wang \\ School of Electronic and Information Engineering, Tianjin University, Tianjin 300072, China \\ Correspondence should be addressed to Jinsheng Yang, jsyang@tju.edu.cn
}

Received 29 August 2011; Accepted 2 October 2011

Academic Editor: Yan Zhang

Copyright () 2012 Jinsheng Yang et al. This is an open access article distributed under the Creative Commons Attribution License, which permits unrestricted use, distribution, and reproduction in any medium, provided the original work is properly cited.

\begin{abstract}
Recently, the scatter cluster models which precisely evaluate the performance of the wireless communication system have been proposed in the literature. However, the conventional SAGE algorithm does not work for these scatter cluster-based models because it performs poorly when the transmit signals are highly correlated. In this paper, we estimate the time of arrival (TOA), the direction of arrival (DOA), and Doppler frequency for scatter cluster model by the modified multiple signal classification (MUSIC) algorithm. Using the space-time characteristics of the multiray channel, the proposed algorithm combines the temporal filtering techniques and the spatial smoothing techniques to isolate and estimate the incoming rays. The simulation results indicated that the proposed algorithm has lower complexity and is less time-consuming in the dense multipath environment than SAGE algorithm. Furthermore, the estimations' performance increases with elements of receive array and samples length. Thus, the problem of the channel parameter estimation of the scatter cluster model can be effectively addressed with the proposed modified MUSIC algorithm.
\end{abstract}

\section{Introduction}

It is important to estimate the spatial/temporal parameters, such as directions of arrival (DOAs), path delays, frequencies, and so forth, embedded in the receive signals in radar, sonar, and wireless communication systems. It also finds applications in source localization, accident reporting, cargo tracking, and intelligent transportation. For example, a precise estimation of DOAs and frequencies of rays in wireless communication channels can helpfully provide better channel information so as to enhance the performance in terms of coverage, capacity, and quality of service (QoS) considerably and increase resistance against interferences. On the other hand, an effective channel model must rely on a realistic characterization of the probability distribution of the relevant channel parameters. So the validation of channel parameter is a prerequisite to ensure that these models reproduce the critical features of the propagation environment, that is, in delay, direction, Doppler, and polarization.

Recently, various high-resolution methods have been proposed in mobile channel to estimate some of the parameters of impinging plane waves, that is, their complex amplitude, relative delay, incidence azimuth, incidence elevation, and Doppler's frequency. These methods can be grouped into three of the categories [1]: spectral estimation, parametric subspace-based estimation (PSBE), and maximum likelihood estimation (ML). The first mentioning category is the MUSIC (multiple signal classification) algorithm [2]. The ESPRIT (estimation of signal parameter via rotational invariance techniques) [3] and unitary ESPRIT [4] methods are included in the PSBE techniques. Among the ML methods, the expectation-maximization (EM) algorithm and the SAGE (space-alternating generalized EM) algorithm [5] have perfect performance. Especially, SAGE algorithm has been applied for joint delay and azimuth estimation in timeinvariant environments as well as for joint delay, azimuth, and Doppler's frequency estimation in time-variant environments.

Unfortunately, the computational burden of the SAGE algorithm is high due to the necessity of nonlinear and multidimensional optimization procedure. Since Swindlehurst proposed several computational efficient algorithms for the estimation of the delays of a multiray channel and solved the spatial signatures (or DOAs) as a least square problem [6], 
many algorithms which can estimate the channel parameters by a $2 \mathrm{D}$ searching on the DOA-delay domain or DOA-frequency domain have been proposed such as JADE-MUSIC algorithm [7], TST-MUSIC algorithm [8], FSF-MUSIC algorithm [9].

With the development understanding of the wave propagation phenomena, the ray model is not suitable to interpret the parameter estimation results of a channel parameter estimator. This is due to the limited resolution of any wireless channel system. A number of radio channel models based on scatter clusters have been proposed in the literature. Many such models which are referred to as cluster delay line (CDL) models simplify the scattering environment and thereby precisely evaluate the performance of the communication system.

However, the SAGE algorithm did not work for the scatter cluster model because the transmit signals are highly correlated. In this paper, we present a low-complexity, yet highaccuracy, MUSIC-based algorithm, which combines the techniques of temporal filtering and of joint DOA and frequency with two-dimensional (2D) searching. Except for this, there are several other advantages of modified MUSIC algorithm compared with SAGE algorithm, such as the reduction of computation, reduced complexity.

This paper is organized as follows. Section 2 introduces the system model of the fading multiray channels, which assumes the propagation rays to be scatter cluster. In Section 3, we present modified MUSIC algorithm and some related issues. In Section 4, simulation results are presented to verify the performance of the proposed approach. Section 5 comes to the conclusions.

\section{System Model}

We consider a wireless communication system with $M$ closely spaced receive antennas $(R x)$, which is equipped with a uniform linear arrays (ULAs), and all the elements are omnidirectional; the interelement spacing at the receive antennas is half a wavelength. Suppose that the signals have a common center frequency of $f_{0}$, then the corresponding wavelength is $\lambda=c / f$, where $c$ is the speed of propagation. Generally, the radio channel in a wireless communication system is often characterized by a multiray propagation model. According to 3GPP SCM model [10], the received signal at the MS consists of $D$ time-delayed multipath replicas of the transmitted signal. These $D$ paths are defined by the same delays which are caused by scatter clusters, and each path consists of $K_{i}(i=1,2, \ldots, D)$ subpaths. Each subpath represents real ray. The total ray is $K=K_{1}+K_{2}+\cdots+K_{D^{\circ}}$. So, the output of the each element at the receive antennas $(R x)$ can be written as

$$
x(t)=\sum_{d=1}^{D} \sum_{k=1}^{K_{d}} \alpha\left(\theta_{d k}\right) e^{j 2 \pi f_{d k} t} s\left(t-\tau_{d}\right),
$$

where $\alpha\left(\theta_{d k}\right)=\exp \left\{j 2 \pi d_{m} \sin \left(\theta_{d k}\right) / \lambda\right\}$ denotes the $m$ th receive antenna response to the ray from $\theta_{d k}$, in which $\theta_{d k}$ is the DOA of the $k$ th subpath of the $d$ th path. $f_{d k}$ denotes Doppler's frequency of the $k$ th subpath of the $d$ th path. If a narrow-band transmit signal and a slow-fading environment are considered, we can assume that the signals are block fading; that is, $s\left(t-\tau_{d}\right)$ remains unchanged in a short period and fades independently from block to block. After appending $T$ time samples at the $m$ th receive antennas, we can obtain the block signals in matrix:

$$
X(t)=A(\theta) \operatorname{diag}(s(t-\tau)) B^{T}(f)+N(t) .
$$
yields

Using the general relation, $\operatorname{vec}(A \operatorname{diag}[b] C)=(A \diamond C T) b$,

$$
X(t)=A(\theta) \diamond B\left(f_{k}\right) \cdot S(t-\tau)+N(t)
$$

where $X(t)=\left[x\left(t_{m}\right), x\left(t_{m}+T_{s}\right), \ldots, x\left(t_{m}+(T-1) T_{s}\right)\right]$, with $T_{s}$ denoting the sampling period and $x\left(t_{m}\right)=\left[x_{1}\left(t_{m}\right), \ldots\right.$, $\left.x_{M}\left(t_{m}\right)\right]^{T}$. Then the Khatri-Rao product $A(\theta) \diamond B(f) \in$ $C^{M L \times K}$, and $N(t)$ is an additive noise process, assumed to be a zero-mean Gaussian noise vector with covariance $\sigma^{2} I$. The receive array response matrix $A(\theta)=\left[A_{1}(\theta), \ldots, A_{D}(\theta)\right]$, $A_{d}(\theta)=\left[\alpha_{1}(\theta), \ldots, \alpha_{K i}(\theta)\right]$, where $\alpha\left(\theta_{i}\right)$ denotes the array response vector of the $i$ th ray:

$$
\alpha\left(\theta_{i}\right)=\left[1, \exp \left\{j \pi \sin \left(\theta_{i}\right)\right\}, \ldots, \exp \left\{j \pi(M-1) \sin \left(\theta_{i}\right)\right\}\right]^{T} .
$$

The frequency array response matrix $B(f)$ is $L$ frequency samples in a short period, with $f_{s}$ denoting the sampling rate, $B(f)=\left[B\left(f_{1}\right), \ldots, B\left(f_{D K}\right)\right]$ :

$$
B\left(f_{k}\right)=\left[1, \exp \left(\frac{j 2 \pi f}{f_{s}}\right), \ldots, \exp \left(\frac{j 2 \pi(L-1) f}{f_{s}}\right)\right]^{T},
$$

where $S(t-\tau)=\left[s\left(t-\tau_{1}\right), \ldots, s\left(t-\tau_{D}\right)\right]^{T}$ denotes the transmit signal which has a delay $\tau$.

\section{Modified MUSIC Algorithm}

Because the rank of correlation matrix is the number of cluster and not the number of ray, the conventional MUSIC algorithm does not work for scatter cluster model directly. So we make some modification based on the conventional MUSIC algorithm. The flow chart of the modified MUSIC algorithm is illustrated in Figure 1.

3.1. The Temporal Filter Method. Despite the conventional MUSIC algorithm constructs a spatial correlation matrix, the proposed algorithm is based on the decomposition of the theoretical temporal correlation matrix $R^{t}$ into a signal subspace $E_{s}$ and a noise subspace $E_{N}$ :

$$
R^{t}=E\left\{X^{H}(t) \cdot X(t)\right\}=S^{H} B_{S} S+\sigma^{2} I .
$$

Note that the number of the incoming paths is assumed to be known a priori; otherwise, we may estimate $D$ by thresholding the magnitude of the eigenvalues of the covariance matrices involved or by using the AIC and the MDL 


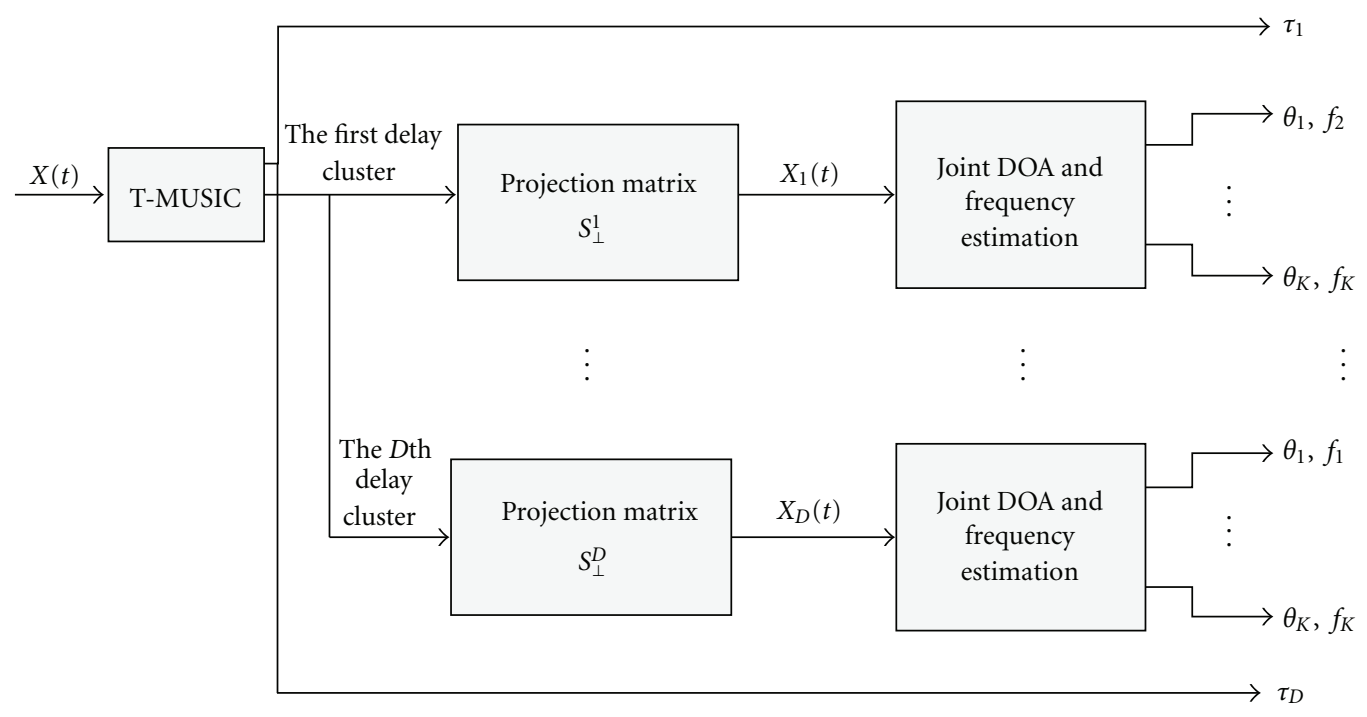

FIgURE 1: The flow chart of the our modified MUSIC algorithm.

detection methods [11], then the next step is similar to the conventional MUSIC algorithm:

$$
R^{t}=V_{s}^{t} \Lambda_{s}^{t} V_{s}^{t H}+V_{n}^{t} \Lambda_{n}^{t} V_{n}^{t H} .
$$

The column vectors of $V_{s}^{t}$ are the eigenvectors that span the signal subspace $R_{s}$, respectively, corresponding to the $D$ largest eigenvalues. The column vectors of $V_{N}^{t}$ spanned by the rest of the and T-D eigenvectors of $R^{t}$ are the orthogonal complement of the column vectors of $V_{s}^{t}$.

Using the orthogonality property between the signal and the noise subspaces, the T-MUSIC algorithm estimates the path delays by

$$
\hat{\tau}=\arg \min \widetilde{s}(\tau)^{H}\left(I-V_{s}^{t} V_{s}^{t H}\right) \widetilde{s}(\tau) .
$$

After searching $\tau$ over the range of interest, the spectrum of the T-MUSIC is, respectively, defined as

$$
P_{\text {MUSIC }}^{t}(\tau)=\frac{1}{\widetilde{s}(\tau)^{H}\left(I-V_{s}^{t} V_{s}^{t H}\right) \tilde{s}(\tau)} .
$$

By applying the T-MUSIC, the resulting delays are estimated. Based on the delay estimates, we define the temporal filtering matrices as

$$
S_{\perp}=I-s(\tau) \cdot\left(s(\tau)^{H} s(\tau)\right)^{-1} \cdot s(\tau)^{H} .
$$

Note that $S_{\perp}$ is the complement project matrix of $s(\tau)$ with $s(\tau)^{H} \cdot S_{\perp}=0^{T}$; with these facts, the modified MUSIC algorithm postmultiplies $S_{\perp}$ to $X(t)$, which is referred to as the temporal filtering process to separate the rays with different delays. Then the output of the $d$ th temporal filter is given by

$$
X_{d}(t)=X(t) \prod_{i=1, i \neq d}^{D} S_{\perp}^{i} .
$$

3.2. Spatial Smoothing (SS) Method. After applying the temporal filter, we can jointly estimate DOAs and Doppler frequencies of each delay cluster. However, the signals included a delay cluster are coherent, the matrix becomes singular, so that some of its eigenvalues are zero. This means that part of the signal subspace is indistinguishable from the noise subspace. As a result, the observed noise subspace is no longer orthogonal to the signal subspace and the MUSIC algorithm fails. So, to overcome these problems, we use a technique called spatial smoothing (SS) to allow the MUSIC algorithm to be applied to the coherent signal case [12].

The basic idea is to form covariance matrices from subsets of the array, which is equivalent to partitioning the original covariance matrix. If there are $P$ subarrays, each subarray is of size $L=M-P+1$ and the output of forward subarray is denoted by $X_{p}^{f}(t)$ with elements $\left[x_{p}(t), \ldots\right.$, $\left.x_{p+L-1}(t)\right]$.

Then we can jointly estimate DOAs and frequencies in one delay cluster by the spatial correlation matrix $R^{s}$, where

$$
R^{s}=\frac{1}{P} E\left\{X_{p}^{f}(t) X_{p}^{f}(t)^{H}\right\} .
$$

Similarly, after eigen decomposition of $R^{s}$, the eigenvectors can be divided into two groups: eigenvectors corresponding to the largest $K$ eigen values are called signal eigenvectors and the subspace they span is called signal subspace. Utilizing the theory of orthogonality, we can obtain the spectrum:

$$
P_{\text {MUSIC }}(\theta, f)=\frac{1}{(a(\theta) \otimes b(f))^{H}\left(I-V_{s}^{t} V_{s}^{t H}\right)(a(\theta) \otimes b(f))} .
$$

\section{Simulation Analysis}

In this section, we conduct simulations to assess the proposed MUSIC algorithm. Assume narrow-band signals that 
are transmitted through 7 rays and received by a ten-element $(M=10)$ uniform linear array, the spacing of two elements is half a wavelength. The 7 rays are divided into two groups: 4 rays have the same delay of $20 \mu \mathrm{s}$, the remains are $40 \mu \mathrm{s}$ delay; the first delay cluster: $\left(80^{\circ}, 30 \mathrm{kHz}\right),\left(60^{\circ}, 60 \mathrm{kHz}\right)$, $\left(40^{\circ}, 90 \mathrm{kHz}\right),\left(50^{\circ}, 90 \mathrm{kHz}\right)$ and the second delay cluster: $\left(20^{\circ}, 120 \mathrm{kHz}\right),\left(45^{\circ}, 30 \mathrm{kHz}\right),\left(15^{\circ}, 60 \mathrm{kHz}\right)$. We sample the receive signal $T$ times, $T=512$. In each short sample, we also have $L=3$ frequency samples, each signal's SNR is set to be $20 \mathrm{~dB}$, and the additive Gaussian white noise (AWGN) is considered.

Figure 2 indicates the two delay clusters estimation of the synthetic channel. Each spectrum peak means Each delayed scatter cluster. The different cluster with $20 \mu \mathrm{s}$ and $40 \mu \mathrm{s}$ delay can be isolated directly. Figure 3 only shows the joint DOA and frequency estimation of the first delay cluster. Each spectrum peak indicates each incoming ray which is included the first delayed cluster with the delay of $20 \mu \mathrm{s}$. All of the group and the single ray can be isolated by the temporal filtering process and the spatial smoothing process during the procedure of the proposed algorithm.

As illustrated in Figures 2 and 3, the modified MUSIC algorithm we proposed could fulfill estimation with high resolving capability for the scatter cluster models.

4.1. Complexity Analysis. In this part, we hereby make a brief comparison for the algorithmic costs in contrast with the modified MUSIC algorithm and SAGE algorithm. Table 1 gives the comparison of complexity for these algorithms.

When the number of ray estimated is small, it is proved that the SAGE algorithm can achieve coverage within 6-10 iterations through simulation analysis. The complexity of SAGE algorithm is less than that of the modified MUSIC algorithm.

On the contrary, the SAGE algorithm achieves coverage within 20 iterations when the wireless scatter environment is dense multipath. So, the complexity of SAGE algorithm is larger than that of the modified MUSIC algorithm.

From Table 1, it is clearly indicated that modified MUSIC algorithm can be optimal in the dense multipath wireless environment among the methods that we have investigated.

4.2. RMSE Analysis for Specular Model. To compare the estimation performance of both algorithms, we present 300 Monte Carlo's simulations to assess the angle estimation performance of our algorithm in specular model and define root mean squared error (RMSE) as

$$
\frac{1}{K} \sum_{k=1}^{K} \sqrt{\frac{1}{300} \sum_{n=1}^{300}\left(\hat{\theta}_{k, n}-\theta_{k}\right)^{2}}
$$

where $\theta_{k, n}$ is the estimate of DOA $\theta_{k}$ of the $n$th Monte Carlo trial. Figure 4 shows the DOA estimation performance comparison with modified MUSIC algorithm and SAGE algorithm in specular models. It is indicated that the performances of angle estimation are nearly the same with modified MUSIC algorithm and SAGE algorithm. However,

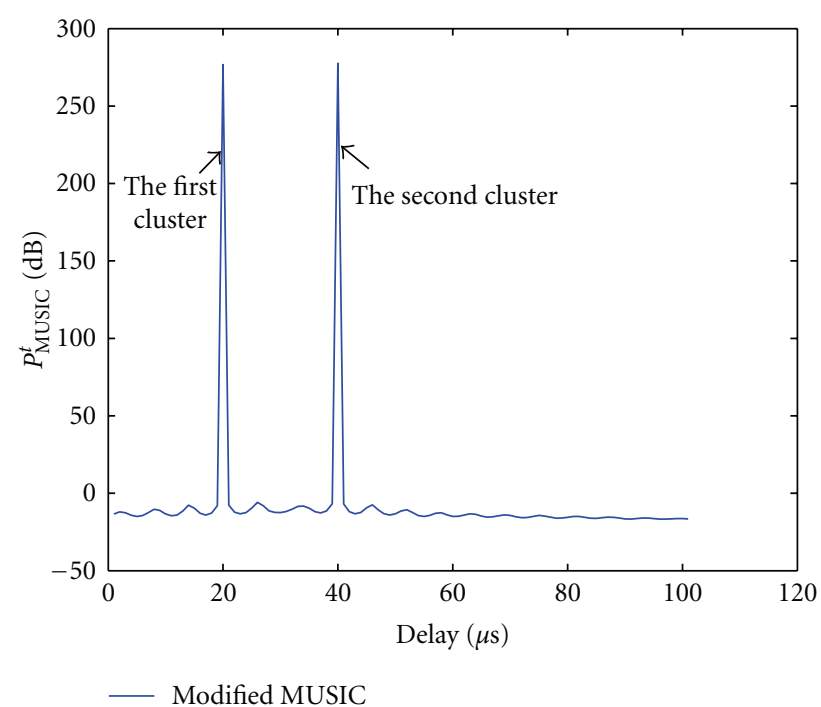

Figure 2: Delay clusters estimation using our algorithm.

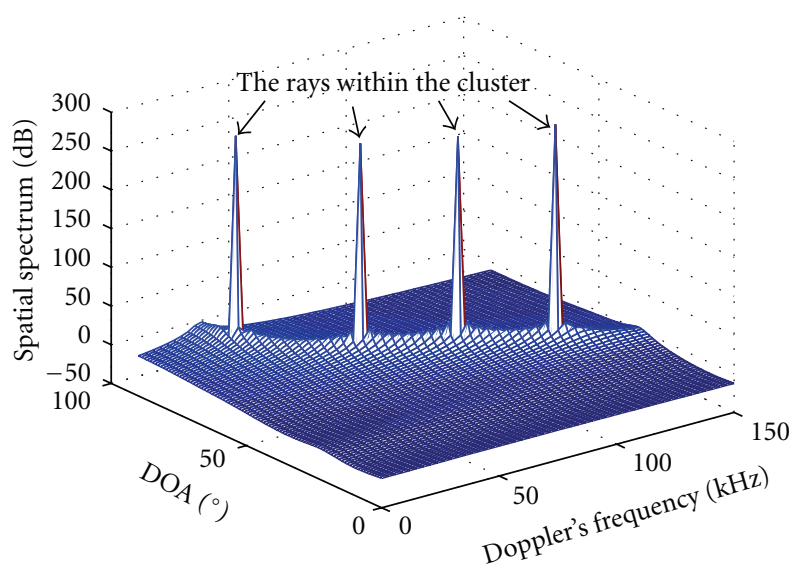

Figure 3: Joint DOA and Doppler's frequency estimation of first cluster using our algorithm.

the modified MUSIC algorithm takes less time than the SAGE algorithm in dense multipath environment.

4.3. RMSE Analysis for Cluster Model. Through the simulation, it is found that the iteration step shows sharp fluctuation for scatter cluster model using the SAGE algorithm. The major reason which leads to the failure of SAGE algorithm in the scatter clusters model is the searching procedure in the $M$ step of SAGE algorithm that does not identify the peak caused by the signals which have same delays.

As mentioned above, the modified MUSIC algorithm is proved to be applied for the cluster models. However, the estimation performance depends on the mean of correlation matrix which substitutes for the expect of the correlation matrix in the practice. So, the number of receive antennas and sample length make a great part in the accuracy of estimation.

Figure 5 illustrates the DOA estimation performance comparison with different elements of receive array. It is 
TABLE 1: The complexity for algorithms.

\begin{tabular}{ll}
\hline Algorithm & Complexity \\
\hline Modified MUSIC algorithm & $T^{2} \times N \times L+O\left(T^{2}\right)+T \times O(T)+(N \times L)^{2} * T+O\left((N * L)^{2}\right)+n 1 * n 2 *\left((N \times L)^{2}+(P \times L)\right)$ \\
SAGE algorithm & The number of iteration $\times$ the number of ray estimated $\times(T * N * T+n 1 * N * T+n 2 * N * T)$ \\
\hline
\end{tabular}

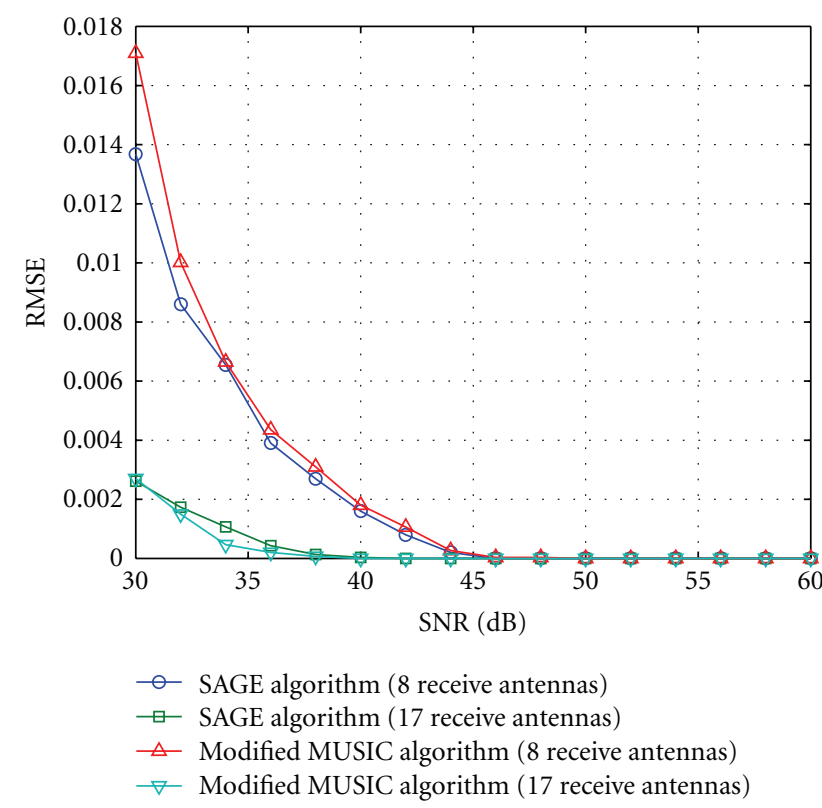

FIgURE 4: The RMSE of DOA estimation performance with Modified MUSIC algorithm and SAGE algorithm in specular models.

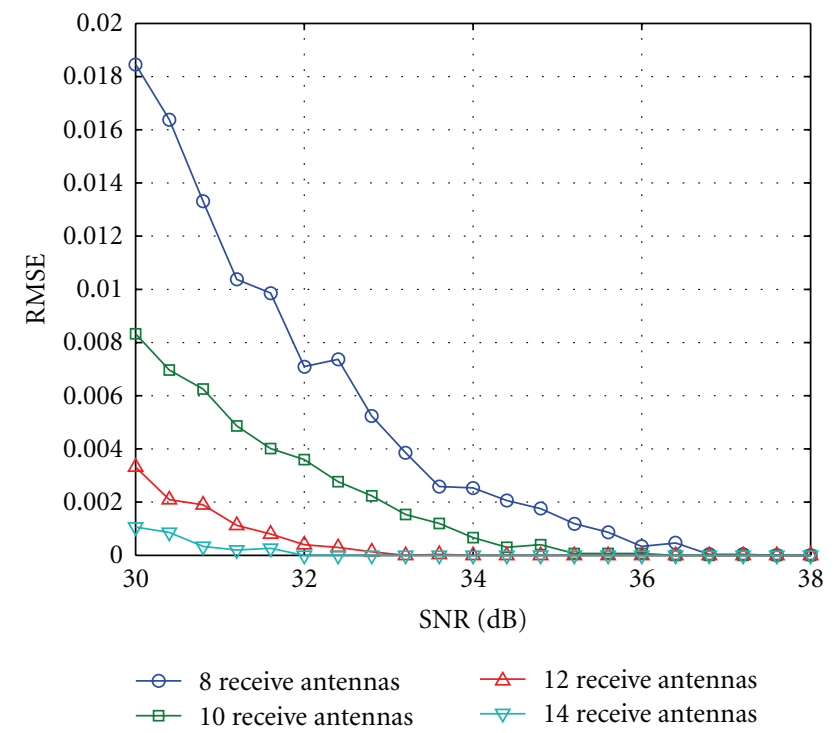

FIGURE 5: The DOA estimation performance with different elements of receive array.

clearly shown that the angle estimation performance of our algorithm is gradually improving with the number of antennas increasing. Multiple antennas improve the estimation performance because of diversity gain. Figure 6 shows the DOA estimation performance comparison with different

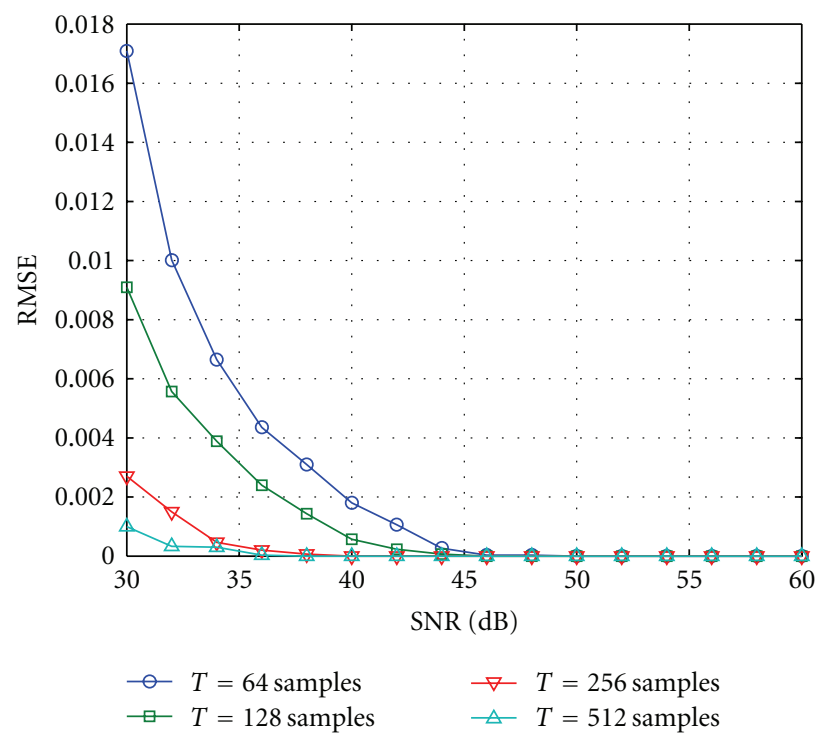

Figure 6: The DOA estimation performance with different samples.

samples. The element of receive array of 8-elements was assumed in this simulation. We confirm that the performance of angles estimation becomes better in collaboration with the increasing samples rate.

\section{Conclusions}

As to the conventional SAGE algorithm does not work for these scatter cluster models, in this paper a modification channel parameter method was proposed which combines the temporal filtering techniques and the spatial smoothing techniques based on the conventional MUSIC algorithm. The synthetic channel was then adopted in simulation to evaluate its performance. We come to a conclusion that the modified MUSIC algorithm takes less time than the SAGE algorithm and has less complexity than the SAGE algorithm in dense multipath environment. The results indicated that this method could fulfill parameter estimation with high resolving capability. It is demonstrated that the estimation performance grows with the number of array elements and the sample rate.

\section{Acknowledgments}

This work is supported by the National Science and Technology Major Project of the Ministry of Industry and Information of China (2009ZX03007-003) and the National Natural Science Foundation of China (61101223). 


\section{References}

[1] H. Krim and M. Viberg, "Two decades of array signal processing research: the parametric approach,” IEEE Signal Processing Magazine, vol. 13, no. 4, pp. 67-94, 1996.

[2] R. O. Schmidt, "Multiple emitter location and signal parameter estimation," IEEE Transactions on Antennas and Propagation, vol. 34, no. 3, pp. 276-280, 1986.

[3] R. Roy and T. Kailath, "ESPRIT_estimation of signal parameters via rotational invariance techniques," IEEE Transactions on Acoustics, Speech, and Signal Processing, vol. 37, no. 7, pp. 984-995, 1989.

[4] M. Haardt and J. A. Nossek, "Unitary ESPRIT: how to obtain increased estimation accuracy with a reduced computational burden," IEEE Transactions on Signal Processing, vol. 43, no. 5, pp. 1232-1242, 1995.

[5] X. Yin, L. Liu, D. K. Nielsen, T. Pedersen, and B. H. Fleury, "A SAGE algorithm for estimation of the direction power spectrum of individual path components," in Proceedings of the 50th Annual IEEE Global Telecommunications Conference (GLOBECOM '07), pp. 3024-3028, November 2007.

[6] A. L. Swindlehurst, "Time delay and spatial signature estimation using known asynchronous signals," IEEE Transactions on Signal Processing, vol. 46, no. 2, pp. 449-462, 1998.

[7] I. Jaafar, R. Amara, H. Boujemâa, and M. Siala, "Joint angle and delay estimation of point sources," in Proceedings of the 12th IEEE International Conference on Electronics, Circuits and Systems (ICECS '05), pp. 1-5, December 2005.

[8] Y. Y. Wang, J. T. Chen, and W. H. Fang, "TST-MUSIC for joint DOA-delay estimation," IEEE Transactions on Signal Processing, vol. 49, no. 4, pp. 721-729, 2001.

[9] J. D. Lin, W. H. Fang, Y. Y. Wang, and J. T. Chen, "FSF MUSIC for joint DOA and frequency estimation and its performance analysis," IEEE Transactions on Signal Processing, vol. 54, no. 12, pp. 4529-4542, 2006.

[10] 3GPP, "Spatial channel model for multiple input multiple output MIMO simulations," Technical Specification Group Radio Access Network TR25.996 v6.1.0, 3GPP, September 2003.

[11] B. Nadler, "Nonparametric detection of signals by information theoretic criteria: performance analysis and an improved estimator," IEEE Transactions on Signal Processing, vol. 58, no. 5, pp. 2746-2756, 2010.

[12] J. S. Thompson, P. M. Grant, and B. Mulgrew, "Performance of spatial smoothing algorithms for correlated sources," IEEE Transactions on Signal Processing, vol. 44, no. 4, pp. 1040-1046, 1996. 

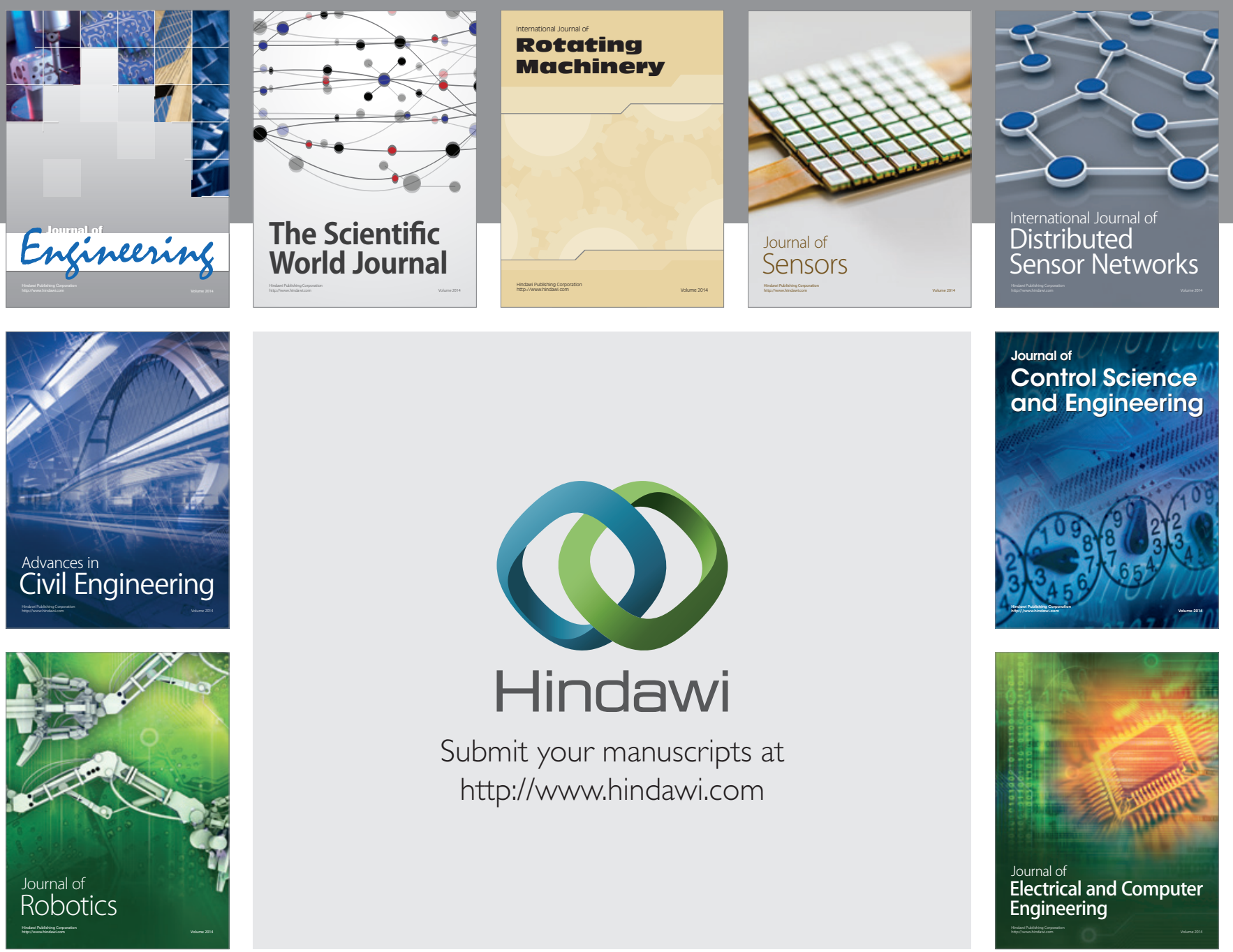

Submit your manuscripts at

http://www.hindawi.com
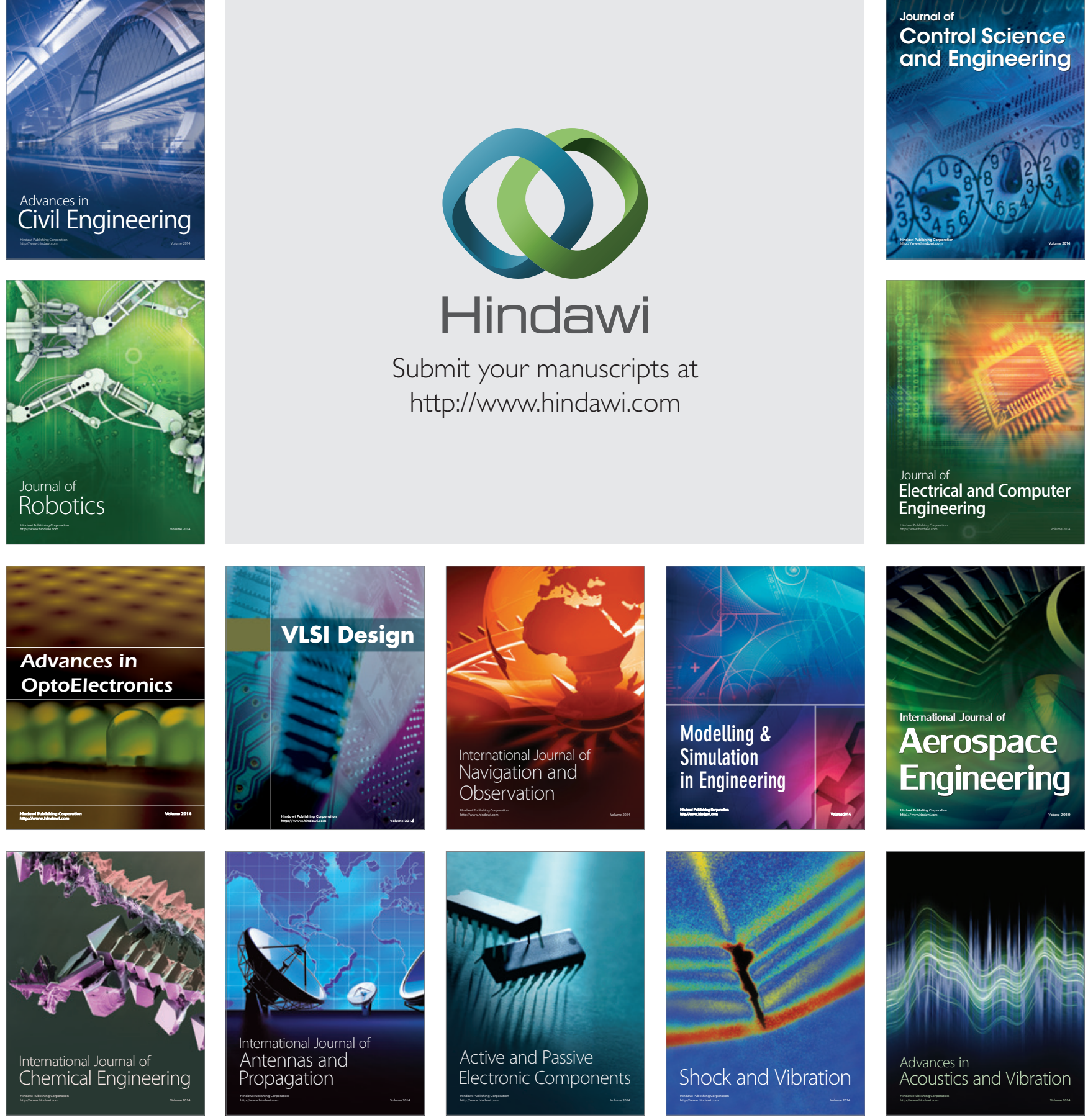